Der Beitrag von Prof. Valerie Junod berichtet über eine wichtige Praxisänderung des Bundesgerichts. Das neue Urteil verbessert die Waffengleichheit für den angegriffenen Arzt deutlich. Er muss für seine Verteidigung nicht mehr im Nebel stochern wie bisher.

Unbefriedigend bleibt, dass der Arzt immer noch die Beweislast dafür trägt, dass er andere Patienten hat als seine Durchschnittskollegen. Dabei zeigt die Managed-Care-Literatur in den USA seit vielen Jahren, dass die Patienten typischerweise gerade nicht homogen auf die Ärzte einer Fachrichtung verteilt sind [1]. Ohne statistische Berücksichtigung der Morbidität sind diese Verfahren deshalb immer noch eine zynische Aufforderung an den Arzt, seine Patienten nach ihrem Risiko zu selektionieren.

Dass die Durchschnittskostenmethode und der ANOVA-Index, so wie sie heute von santésuisse praktiziert werden, aus rechtlicher und statistischer Sicht an wesentlichen Mängeln leiden und sich nicht eignen, um unwirtschaftliches Handeln der Ärzte nachzuweisen, zeigt auch deutlich der Artikel von Dr. Michel Romanens. Er fasst die wesentlichen Erkenntnisse aus drei vom Verein Ethik und Medizin in Auftrag gegebenen rechtlichen und statistischen Gutachten zusammen, nennt die neuste Rechtsprechung des Bundesgerichts zum Wirtschaftlichkeitsverfahren und wirft einen Blick in die Zukunft.

An santésuisse geht das Angebot, die WZW-Verfahren gemeinsam mit der FMH auf eine neue Basis zu stellen. Und ans Parlament geht die Aufforderung, Art. 56 KVG zu ergänzen mit dem Ziel, die wichtige Aufgabe den Kassen und Leistungserbringerverbänden gemeinsam zu übertragen, sachgerechte und damit eines Rechtsstaats würdige Wirtschaftlichkeitsverfahren zu entwickeln.

Hanspeter Kuhn, Fürsprecher; Gabriela Lang, Rechtsanwältin; Rechtsdienst FMH

1 Siehe statt vieler: Kongstvedt PR. Essentials of Managed Health Care. $4^{\text {th }}$ edition. Gaithersburg: MD Aspen Publication; 2001. S. 400: Peer group, adjusted for age, sex and case-mix/severity of illness - «the issue of severity must be addressed».

\title{
Wirtschaftlichkeitsverfahren: endlich eine gute Nachricht
}

\section{Valerie Junod}

Professorin an der juristischen Fakultät der Universitäten Genf und Lausanne

Die Autorin bedankt sich bei Betty und Charles-André Junod für ihre kritischen Kommentare.

Korrespondenz:

Prof. Dr. iur. Valerie Junod Universität Lausanne - HEC Internef bureau 615 CH-1015 Lausanne Tel. 0216923428 Fax 0216923305

valerie.junod@unil.ch
Ein Urteil des Bundesgerichts war kurz vor Weihnachten Balsam für die Seele der Ärzte [1]. Der Entscheid begrenzt die Macht der Kassen und des Branchenverbands santésuisse, die (Un-)Wirtschaftlichkeit der Angehörigen der Gesundheitsberufe [2], insbesondere natürlich der Ärzte, zu kontrollieren und zu sanktionieren.

Schon seit 1969 verlangen die Krankenkassen von den Ärzten die Rückzahlung der aus Kassensicht infolge sogenannter Überarztung «zu viel» gezahlter Beträge. Die Berechnung des eingeforderten Betrags basiert auf dem Durchschnittsvergleich mit den Kollegen desselben Fachgebiets und derselben Region [3]. Nach der Rechtsprechung wird eine Toleranzbreite von rund 30\% gewährt. Ein Beispiel: Der Kardiologe aus Lausanne, der seinen Krankenversicherungs-

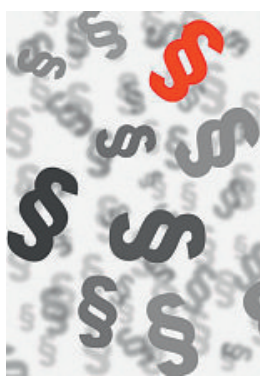
patienten direkte (Behandlungen) und/oder indirekte Kosten (insbesondere Medikamente und Analysen) verrechnet, die pro Patient und Jahr mehr als 30\% höher sind als die Durchschnittskosten seiner Waadtländer Kardiologenkollegen, riskiert, zur Zahlung der Differenz aufgefordert zu werden [4]. Um der Anwendung dieser sogenannten «statistischen» Methode zu entgehen, kann der Arzt versuchen, objektive Rechtfertigungsgründe vorzubringen, beispielsweise die besondere Zusammensetzung seiner Patienten. Doch liegt es an ihm, dafür den vollen Beweis zu erbringen.

Nach einer oder mehreren Warnungen können die Kassen, die sich auf die Statistik von santésuisse stützen, eine Forderungsklage vor dem kantonalen Gericht einreichen. Im Jahr 2007 waren über die ganze Schweiz verteilt rund 40 solche Prozesse hängig [5].

Im vorliegenden Fall war ein Genfer Allgemeinpraktiker dazu verurteilt worden, rund 300000 Franken $\mathrm{zu}$ bezahlen. In der Beschwerde gegen diesen Entscheid des kantonalen Schiedsgerichts hat der Arzt verschiedene Kritikpunkte angeführt. Die meis-

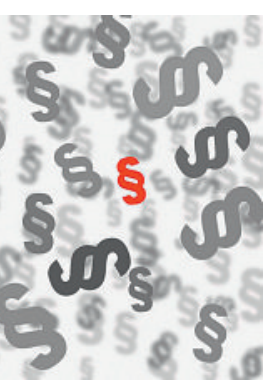
ten wurden kurz und ziemlich sec abgewiesen [6].

Eine Kritik hat das Bundesgericht aber akzeptiert, und diese hat eine grosse praktische Bedeutung. Der Arzt rügte eine Verletzung seiner verfassungsmässigen Rechte (Art. 29 Bundesverfassung) und der europäischen Menschenrechtskonvention (Art. 6 EMRK). Diese Bestimmungen garantieren das rechtliche Gehör. Konkret rügte der Arzt, dass die Zahlen, auf die sich das kantonale Urteil gestützt habe, so undurchsichtig gewesen seien, dass er seine Verteidigungsrechte nicht wirksam habe geltend machen können.

Es ist erhellend, sich in diesem Zusammenhang in Erinnerung zu rufen, dass santésuisse dem Richter den Betrag der vom angegriffenen Arzt verursachten Kosten und den Durchschnittswert der Ärzte aus der Vergleichsgruppe mitteilt. In Ergänzung zu dieser 
Durchschnittsangabe erhält der Richter die Anzahl der Ärzte aus der Vergleichsgruppe und das Durchschnittsalter der von ihnen behandelten Patienten. Das Bundesgericht kommt zum Schluss, dass die Angaben zur Vergleichsgruppe zu lückenhaft waren, um vom angegriffenen Arzt verstanden, kontrolliert

\section{Das Urteil eröffnet dem Arzt die Möglichkeit, seine nominell höheren Durchschnittskosten viel konkreter mit denen seiner Kollegen zu vergleichen.}

und kritisch hinterfragt werden zu können. Das Bundesgericht verlangt deshalb einen weitergehenden Zugang («un accès plus étendu»).

Was bedeutet das? Das Bundesgericht präzisiert in zwei Punkten:

«Einerseits erlaubt erst die Kenntnis der Namen der Ärzte der Vergleichsgruppe zu verifizieren, ob darunter Ärzte sind, die zu einer anderen Gruppe gehören oder ob Ärzte nicht in der Vergleichsgruppe sind, die in sie aufgenommen hätten werden müssen. Andererseits erlaubt erst die anonymisierte Kenntnis der Verteilung der Kosten für jeden Arzt der Vergleichsgruppe, also die anonymisierte Kenntnis derselben Daten, wie sie santésuisse für den angegriffenen Arzt mitteilt («Datenpool von santésuisse»), dem angegriffenen Arzt, sich konkret mit seinen Kollegen zu vergleichen und eine gezielte und wirksame Verteidigung wahrzunehmen») [7] (Übersetzung FMH).

Zusammengefasst verlangt das Bundesgericht von santésuisse, dem angegriffenen Arzt die Identität der Ärzte aus der Vergleichsgruppe bekannt zu geben. Der Dachverband der Krankenversicherer muss zudem für jeden von ihnen die zu den Daten des angegriffenen Arztes äquivalenten Zahlen bekanntgeben. Konkret bedeutet dies für die direkten Kosten: die Kosten der durchgeführten Behandlungen und der abgegebenen Medikamente, die Anzahl der Besuche und Konsultationen; das Durchschnittsalter und die Zahl der behandelten Patienten; das Total und die abgeleiteten Indices der vorangegangenen Zahlen [8]; für die indirekten Kosten: die Kosten der verschriebenen Medikamente, Analysen und Physiotherapiesitzungen; auch hier die Totale und die daraus abgeleiteten Indices [9].

Zweifellos ist dieses Urteil für den angegriffenen Arzt eine sehr erfreuliche Nachricht. Es eröffnet ihm die Möglichkeit, seine nominell höheren Durchschnittskosten viel konkreter mit denen seiner Kollegen zu vergleichen. So kann er zum Beispiel zeigen, dass einige Ärzte aus der Vergleichsgruppe nicht oder nicht mehr in derselben Spezialität tätig sind, und es wird ihm viel eher möglich sein, Besonderheiten seiner Patienten im Vergleich zu den Patienten der verglichenen Arztkollegen darzulegen. Mit dem Urteil wurde kein Allheilmittel geschaffen, aber die
Länge der Spiesse der involvierten Parteien hat eine signifikante Annäherung erfahren, was als deutliche Verbesserung gewertet werden kann.

Für die betroffenen Ärzte aus der Vergleichsgruppe kann diese Transparenz unangenehm sein. Es ist nicht ausgeschlossen, dass der angegriffene Arzt aus den in Zukunft verlangten Daten das Einkommen seiner Kollegen ableiten kann [10]. Das Bundesgericht hat sich nicht dazu geäussert, wie das rechtliche Gehör mit dem Schutz der Privatsphäre und dem Datenschutz der betroffenen Kollegen vereinbart werden kann.

Schliesslich bedeutet das Urteil für die Kassen, dass sie die zur Begründung einer Unwirtschaftlichkeit verwendeten Daten nicht mehr für sich behalten können. Wenn sie die Verurteilung eines Arztes erreichen wollen, müssen sie eine deutlich verbesserte Transparenz gewähren. Gut möglich, dass sich mit der Zeit der ganze Ablauf der Wirtschaftlichkeitsverfahren ändern wird.

\section{Literatur}

1 Entscheid vom 15. Dezember 2010, Referenz 9C_968/2009, auf Internet herunterladbar unter folgender Adresse: http://jumpcgi.bger.ch/cgi-bin/ JumpCG?id=15.12.2010_9C968/2009

2 Ursprünglich wurde der Begriff «Polypragmasie» verwendet. Er wurde mit der Zeit ersetzt durch den Begriff «Wirtschaftlichkeitskontrolle». Diese Kontrolle stützt sich auf Art. 56 des Krankenversicherungsgesetzes. Für eine (französischsprachige) vollständige Darstellung des Kontrollsystems seiner Stärken und Schwächen siehe Junod V. Polypragmasie. Analyse d'une procédure controversée. In: Cahiers genevois et romands de sécurité sociale $\mathrm{n}^{\circ}$ 40-2008. p. 140ss.

3 Die Vergleichsgruppe muss mindestens zehn Ärzte umfassen. Vgl. Erwägungen 5.3.

4 Weil die Rückforderung sich auch auf die indirekten Kosten bezieht, also auf Honorarumsätze, die nicht dem Arzt selbst zukommen, handelt es sich nicht im eigentlichen Wortsinn um eine Rückzahlung.

5 santésuisse. Les évaluations d'économicité de santésuisse; 2009. p. 9.

6 Vgl. Erwägung 2.3 (Verjährungsargument); Erwägung 3.2 (Klageberechtigung und von jeder Kasse verlangter Betrag); Erwägung 6.2 (Infragestellung der statistischen Methode: «Le Tribunal fédéral admet depuis longtemps le recours à la méthode statistique comme moyen de preuve permettant d'établir le caractère économique ou non des traitements prodigués par un médecin donné et n'entend pas modifier sa pratique»).

7 Erwähntes Urteil. Erwägung 6.3.3.

8 Erwähntes Urteil. Erwägung 6.3.2.

9 Id.

10 Die direkten und indirekten Kosten der Vergleichsgruppe müssen individuell, aber anonym mitgeteilt werden. Doch ist es denkbar, dass der angegriffene Arzt sie im Vergleich zur Namensliste der Ärzte aus der Vergleichsgruppe zuweisen kann. Dies ist umso eher möglich, wenn die Vergleichsgruppe klein ist (zur Erinnerung, sie muss im Minimum 10 Ärzte betragen). 